\title{
What can researchers learn from participant involvement? Insights from the ROLO Family Advisory Committee
}

\author{
Eileen O. Brien, Niamh Walsh, Aisling Geraghty, David Byrne and Fionnuala McAuliffe \\ UCD Perinatal Research Centre, School of Medicine, National Maternity Hospital, University College Dublin, Dublin, \\ Ireland, Dublin, Ireland
}

\section{Abstract}

Introduction: Public and patient involvement (PPI) describes how researchers collaborate and engage with the public in order to make research more relevant to them. The ROLO Family Advisory Committee is a self-selected group of parents who are involved in the longitudinal follow-up of the ROLO Study. It was established in 2017 and the goal is to achieve a partnership between ROLO families and researchers, leading to improved research quality, relevance and outcomes. The aim of engaging with parents from the ROLO study is to understand key outcomes of importance for parents relating to their own health and their children's health and to gain insights into how the research agenda can be more relevant to parent and child needs.

Materials and Methods: All parents involved with ROLO study were invited to join the ROLO Family Advisory Committee in 2017. Parents who had expressed interest in joining the committee were invited to attend annual ROLO Families Advisory Committee Meetings in 2018 and 2019. Parents were asked to discuss their opinions about current research projects and potential future projects. This was followed by an open discussion about relevant health questions. These meetings were either transcribed on the day or recorded with audio recordings transcribed verbatim and broad themes identified.

Results: Mothers had greater concern for outcomes relating to their children than themselves. Parents were very interested in understanding the relationship between diet in pregnancy and child health and welcomed research regarding blood tests that could be predictors of future health. Parents had great concern for the effect social media has on child mental health and also wanted more studies conducted in the areas of fussy eating and how to ensure children consume a wide range of healthy foods. It was consistently highlighted that children and teenagers should understand healthy eating principles and ways to promote their physical and mental health.

Discussion: Through the two focus groups, valuable insights were gained regarding what research parents consider relevant and future research questions. The ROLO agenda has certainly been enriched by PPI. We are seeking opportunities to enable research to be carried out in the areas discussed at the meetings.

\section{Conflict of Interest}

There is no conflict of interest 